\title{
Novel Miniature and Broadband Millimeter-Wave Monolithic Star Mixers
}

\author{
Che-Chung Kuo, Chun-Lin Kuo, Che-Jia Kuo, Stephen A. Maas, Fellow, IEEE, and Huei Wang, Fellow, IEEE
}

\begin{abstract}
In this paper, three monolithic star mixers using a new miniature dual balun are proposed. The first one is a double spiral transformer mixer, and the second one is a trifilar transformer mixer. Both of these are fabricated using a commercial GaAs pseudomorphic HEMT process. The third is a 3-D transformer mixer, which is fabricated using a commercial CMOS process. These mixers exhibit bandwidths over $25-45 \mathrm{GHz}(57 \%)$ with local oscillator isolations better than $20 \mathrm{~dB}$. These star mixers are smaller than $(\lambda / 6 \times \lambda / 6)$ for the mixer core area. Compared with traditional star mixers, these mixers demonstrate $80 \%$ size reduction, and achieve good performance with the smallest chip size among all star mixers using monolithic microwave integrated circuit processes.
\end{abstract}

Index Terms-Balun, diode mixer, double-balanced mixer (DBM), mixer, monolithic microwave integrated circuit (MMIC), resistive mixer, spiral transformer, star mixer, transformer.

\section{INTRODUCTION}

A LTHOUGH millimeter-wave (MMW) mixer techniques are well developed, the high-performance and compact monolithic mixer is still a popular research topic. The doublebalanced mixer (DBM), which offers high spur suppression and wide dynamic range, is the most commonly used configuration. Compared to the traditional ring DBM, the advantage of the star DBM is low IF parasitic inductance, which results in wide IF bandwidth. In addition, the IF of the star DBM is dc coupled, allowing for direct conversion of signals to baseband [1]. Therefore, the star DBM is suitable for high data rate, even multigigabit direct-conversion transceiver applications. The traditional structure of the star DBM involves a dual balun, which is not a planar structure [13]. One of the first planar star mixers is shown in Fig. 1. Two Marchand dual baluns were used in this circuit to form a cross arrangement [2]. A modified three-conductor Marchand dual balun with a multicoupled microstrip line balun was reported by Ryu et al. [3]. A lumped miniature dual-balun star mixer in the $S$-band was presented by Chiou $e t$ al. [5]. The star mixers are often impractical in integrated circuit (IC) design because they need two rather large half-wavelength Marchand

Manuscript received July 25, 2007; revised November 22, 2007. This work was supported in part by National Taiwan University under Excellent Research Project 95R0062-AE00-01 and by the National Science Council of Taiwan, R.O.C. under Contract NSC 93-2752-E-002-002-PAE, Contract NSC 93-2219-E-002-024, and Contract NSC 93-2213-E-002-033.

C.-C. Kuo, C.-L. Kuo, C.-J. Kuo, and H. Wang are with the Department of Electrical Engineering and the Graduate Institute of Communication Engineering, National Taiwan University, Taipei 106, Taiwan, R.O.C. (e-mail: hueiwang@ntu.edu.tw).

S. A. Maas is with Nonlinear Technologies Inc., Long Beach, CA 90807 USA.

Digital Object Identifier 10.1109/TMTT.2008.919063

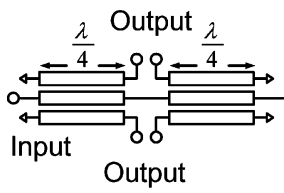

(a)

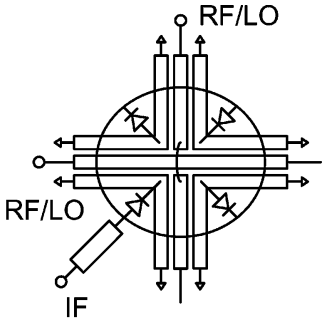

(b)
Fig. 1. (a) Marchand dual balun for star mixer. (b) First planar star mixer reported by Basu and Maas [2].

dual baluns, which increase the chip area. Therefore, the miniature dual balun is a key component of a star mixer.

In this paper, three novel miniature transformer-type Marchand dual baluns are proposed to further reduce the size of the star DBM. The first is a double-spiral transformer. Although spiral transformers are used extensively in monolithic microwave integrated circuit (MMIC) design, we propose a novel double-spiral transformer dual balun for a star mixer. The second is a trifilar transformer. Trifilar transformers are also popular in ICs, but here we propose the use of a dual balun consisting of two trifilar cascaded transformers. These two miniature transformer type Marchand dual baluns are implemented in a commercial GaAs pseudomorphic HEMT (pHEMT) process for star mixers.

Besides, modern CMOS technology with downscaling of feature dimensions, multilayer of interconnected metal, and feasibility of back-end integration becomes an interesting alternative for MMW IC designs. Therefore, we further propose a 3-D structure dual balun for a resistive star DBM using a standard commercial CMOS process. To the authors' knowledge, these mixers (using miniature transformer type Marchand dual baluns) achieve the smallest chip sizes among the published MMIC star mixers in the MMW regime.

\section{Star Mixer CONFIGURATION}

A single-balanced mixer uses $180^{\circ}$ baluns to cancel the local oscillator (LO) signal at RF and IF ports, giving it good LO-to-RF and LO-to-IF isolations [1]. However, RF-to-IF isolation is not very good due to the unbalanced RF signal at the IF port and, thus, a low-pass filter is required to reject the RF signal at the IF terminal. Double-balanced ring mixers avoid this problem by extracting the IF signal from the center tap of the RF balun. This mixer requires an ideal tapped transformer to realize the balun, and the IF connection in a ring mixer is usually difficult to realize using practical microwave baluns [1]. 


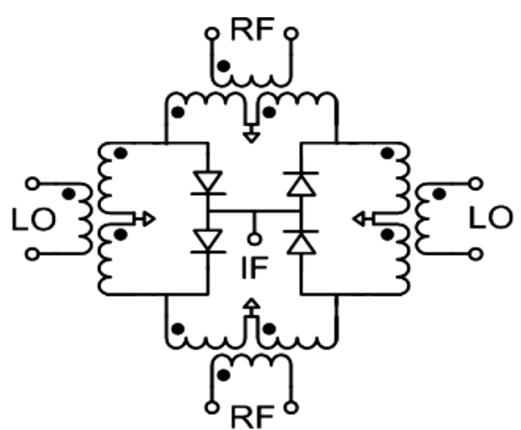

(a)

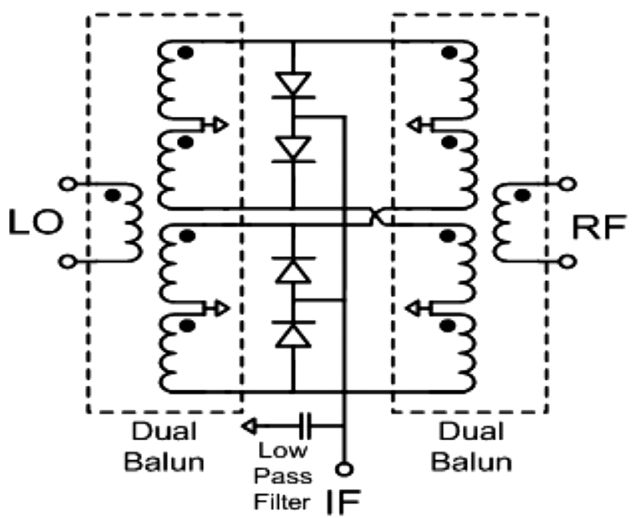

(b)

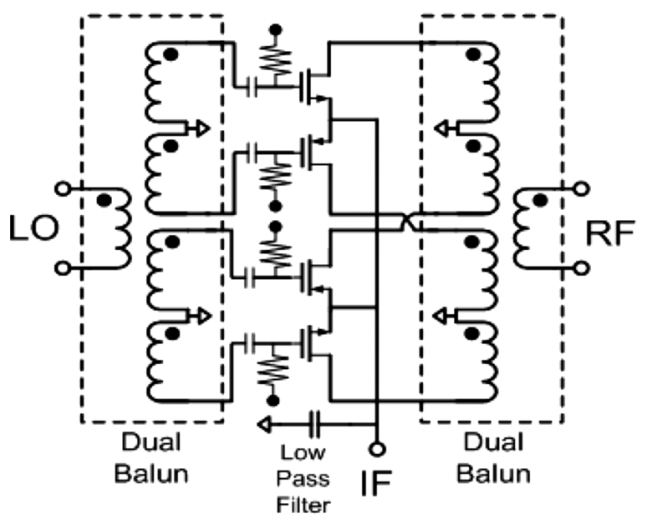

(c)

Fig. 2. Schematics of the: (a) star DBM, (b) star mixer using dual balun, and (c) resistive star mixer using dual balun.

The star mixer shown in Fig. 2(a) overcomes the IF connection problem and has additional advantages as well, including a wide IF bandwidth and dc coupled IF [1]. The four diodes are connected to the common node, which is the IF output. The $\mathrm{RF}$ and LO signals are fed to the diode bridge through a set of transformers, which are often realized as parallel transmission line baluns. The circuit topology in Fig. 2(a) is identical to the traditional star mixer in Fig. 1(b). The circuit structure implementing the novel dual balun proposed in this paper is shown in Fig. 2(b), and the resistive star mixer using CMOS technology is shown in Fig. 2(c).

\section{TRANSFORMERS IN DUAL BALUNS}

To design miniature mixers, three Marchand dual baluns (three-conductor coupled line baluns) are proposed in this paper. These transformers are designed according to the respective characteristics of the GaAs and CMOS MMIC processes. In a typical GaAs process, there are only two metal layers, which are not suitable for multilayer structures and, thus, the transformer must be designed in edge-coupled form. A CMOS process often supports multilayer metals; therefore, the transformer can be designed using broadside-coupled lines in a smaller area.

\section{A. Double Spiral Transformer (Edge Coupled)}

A Marchand dual balun is composed of two series-connected three-conductor coupled-line sets [see Fig. 1(a)]. The two conductor coupled line can be used as a spiral transformer, which is widely used in MMIC design [8], [9], and a three-conductor coupled line spiral transformer, which uses a similar layout, called a double spiral transformer, is shown in Fig. 3(a), where $W$ and $S$ are the metal width and spacing, respectively, and $L A$ is the area of the metal to backside via. The Marchand dual balun [see Fig. 1(a)] is composed of two series-connected double spiral transformers. The crossover lines and ground are realized by air bridges and backside vias, respectively. The double spiral transformer is equivalent to a three-conductor coupled-line transformer. Lines $A$ and $B$ [see Fig. 3(a)] are both a quarter-wavelength long at the desired frequency. For the diode connection shown in the layout of Fig. 2(b), two output ports [ports 2 and 3 in Fig. 3(a)] are needed in the upper and lower sides of the transformer; therefore, these two lines are not wrapped in the same manner. The signal excites port 1 and propagates along line $C$, and the power is coupled to lines $A$ and $B$ due to edge coupling. Line $C$ must be longer than the other lines to ensure that every section of the transformer is formed with three-conductor coupled lines. Port 4 is used for the other cascaded identical transformer. Output port sets 2-2' and $3-3^{\prime}$ are two differential outputs of the dual balun.

\section{B. Tifilar Transformer (Edge Coupled)}

The trifilar balun [see Fig. 3(b)] is also widely used in MMIC designs [8], [10]. Different from the double spiral transformer, the trifilar transformer is a symmetric transformer. Lines $A$ and $B$ also have equal lengths of one quarter-wavelength at the desired frequency. For the diode connection [see Fig. 2(b)], these two lines, which start at the outside turn and go to the inside turn and back to the outside turn repeatedly, are of equal length and wrapped in the same way. Line $C$ must also be somewhat longer than the other two.

\section{3-D Transformer (Broadside Coupled)}

Due to the multilayer dielectric offered in the CMOS process, the 3-D transformer is proposed, as shown in Fig. 3(c). The 3-D dual balun can be divided into three-layer cascaded transformers; every part of the single-layer cascaded transformers is a single spiral transformer. Lines $A$ and $B$ still are a quarter-wavelength long at the desired frequency. As shown in Fig. 3(d), port 1 is the input port in the middle layer, and power is coupled to the upper and lower layers due to broadside coupling. The output signals at the two output port sets $2-2^{\prime}$ and $3-3^{\prime}$ are differential.

There are two benefits of a broadside-coupled transformer compared to the edge-coupled form. First, air bridges are not 


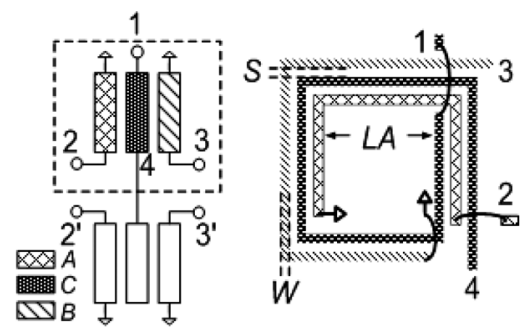

(a)

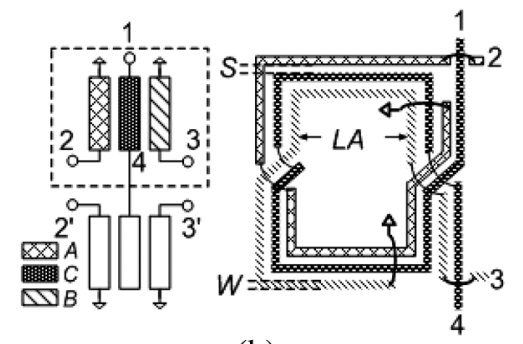

(b)

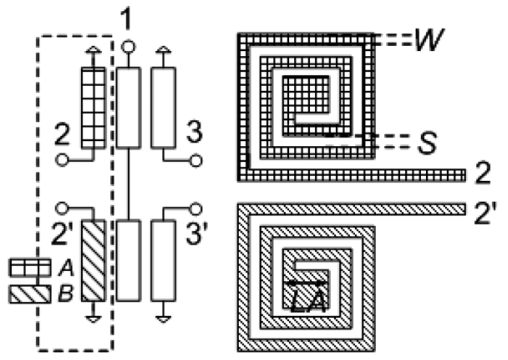

(c)

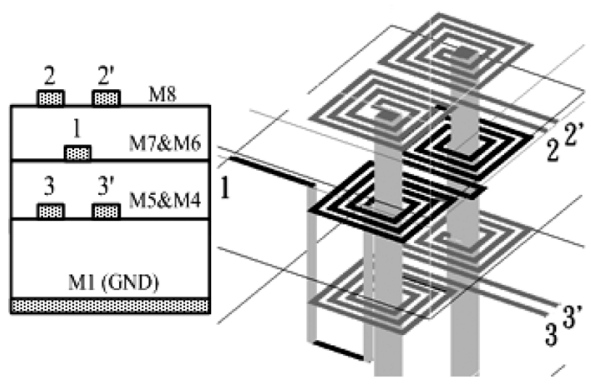

(d)

Fig. 3. Layouts of the: (a) double spiral transformer, (b) trifilar transformer, (c) 3-D transformer, and (d) side view of 3-D transformer dual balun.

needed; the air bridge induces a capacitive parasitic, which can reduce bandwidth. Therefore, a broadside-coupled transformer balun will have a wider bandwidth than that of an edge-coupled transformer. The other benefit is smaller size, which is very obvious.

\section{MARChand Dual BALUn For StAR MiXer}

The star mixer requires two sets of dual baluns for RF and LO signals, respectively. In order to optimize the mixer performance, the even-mode impedance needs to be as high as possible, while the odd-mode impedance is approximately

$$
Z_{o d}=0.5 \sqrt{Z_{d} \times Z_{o}}
$$

where $Z_{d}$ is the diode impedance or input impedance of the transistor, $Z_{0}$ is the source impedance, and $Z_{0 d}$ is the odd-mode impedance of the transformer or balun [2]. Since

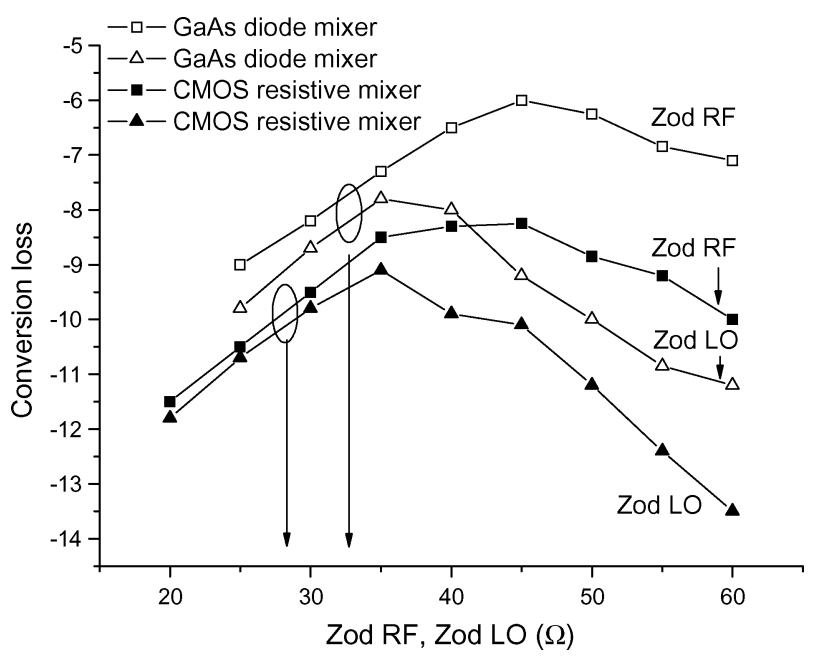

Fig. 4. Computed conversion loss for odd-mode impedances of LO and RF dual baluns. All the lengths in the baluns are a quarter-wavelength at $30 \mathrm{GHz}$.

the even-mode impedance is usually five times higher than the odd-mode impedance in a Marchand balun [1], [2], we therefore only need to consider $Z_{0 d}$ in (1). In the mixer design, the LO or RF balun odd-mode impedance $Z_{0 d}$ can be estimated from the substrate parameters and then the device impedance (diode or MOS) $Z_{d}$ can then be calculated based on (1). However, because the impedance calculation using (1) is for a pair of ideal coupled lines, while the input impedance of the device $Z_{d}$ is complex and will vary under a high-power pump in practical designs, we will suggest using only (1) to estimate the initial required $Z_{d}$ of the device and obtain a range of possible device sizes. After that, we should include the LO drive and the desired baluns to observe the large signal effect in the mixer performance via harmonic balance simulation. Fig. 4 presents the simulated optimal conversion losses versus input impedances of LO and RF dual baluns in GaAs or CMOS processes with the considerations of the device size and LO power. In the simulation, all the lengths of the balun are a quarter-wavelength at $30 \mathrm{GHz}$. Every curve (RF or LO balun) is simulated by varying the odd-mode impedance of the balun and depends on the input impedance of the selected device. Either for the GaAs diode or CMOS, the $Z_{\text {od }}$ of RF and LO baluns to be selected should be at the point where they have a similar conversion loss. Therefore, $Z_{\text {od }}$ of RF and LO baluns to provide the minimum conversion loss suggested in Fig. 4 are approximately 28 and $33 \Omega$ for the baluns in CMOS and GaAs process, respectively.

The GaAs diode mixer is used as an example to explain more clearly how the curves in Fig. 4 were obtained. Firstly, $Z_{0 d}$ was determined to be $33 \Omega$ based on a 5- $\mu$ m metal width on a $100-\mu$ m-thick GaAs substrate. Since $Z_{0}$ is $50 \Omega$, we could have $Z_{d}$ estimated to be $72 \Omega$ from (1). Next we observed the GaAs diode impedance under the LO drive from the simulation and determined that the diodes with the sizes (gatewidth) of $40-120 \mu \mathrm{m}$ have impedances from 60 to $90 \Omega$ under a $5-\mathrm{dBm}$ LO drive. Therefore, we simulated the range of the diode size to produce the curves for optimal conversion loss among the various diode sizes of Fig. 4. We concluded that 7.8-dB conversion 


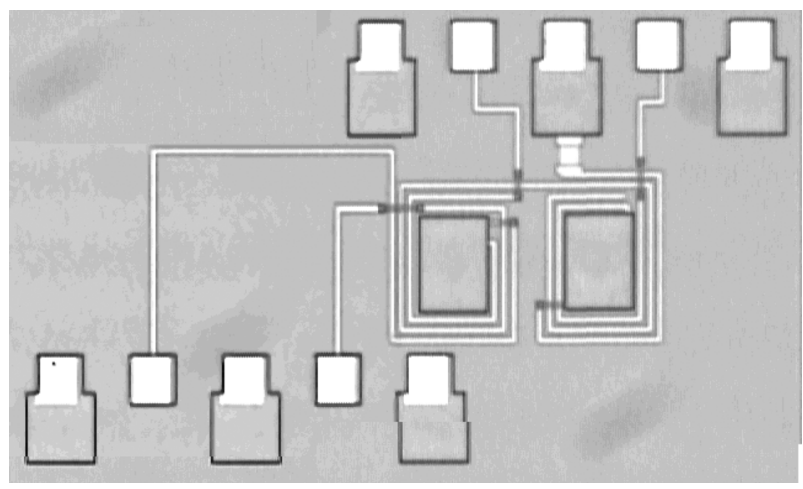

Fig. 5. Chip photograph of the double spiral transformer dual balun. $(0.8 \times$ $0.5 \mathrm{~mm}^{2}$ ).

loss with a diode size of $40 \mu \mathrm{m}$ and $Z_{\text {od }}$ of $32 \Omega$ is the lowest conversion loss achievable in this preliminary estimation, and these parameters can be used for the mixer design. The curves for the CMOS mixer were similarly obtained.

The dual-balun design procedure is summarized as follows:

1) calculate the quarter-wavelength for desired frequency $L$;

2) determine $S$ (metal spacing) and $W$ (metal width) for the appropriate even- and odd-mode impedance from Fig. 4;

3 ) predict $n$ (number of turns);

4) electromagnetic (EM) full-wave software simulation and optimization.

In the design using the GaAs process, $W$ and $S$ are $5 \mu \mathrm{m}$ for maximum even-mode impedance and $L A$ is $60 \times 100 \mu \mathrm{m}^{2}$. The even-mode impedance of $133 \Omega$ and odd impedance of $33 \Omega$ (see Fig. 4) are chosen for optimum performance of the mixer. The center frequency is $40 \mathrm{GHz}$ and performance optimization is achived through EM simulation (Agilent EEsof ADS Momentum). These first two balun circuits are designed using a $0.15-\mu \mathrm{m}$ GaAs pHEMT MMIC process, and wafer probe measurements employ a four-port network analyzer (Anritsu 37397D). The metal lines of RF pads and connections are deembedded in the balun measurement results.

\section{A. Double Spiral Transformer Dual Balun}

In the test circuit of the double spiral transformer dual balun shown in Fig. 5, one port is terminated by a thin-film resistor of $50 \Omega$. The balun size is approximately $400 \times 200 \mu \mathrm{m}^{2}$. The insertion loss of an ideal dual balun is $6 \mathrm{~dB}$, and the simulated and measured insertion loss results are approximately 8 and 8 to $10 \mathrm{~dB}$. The simulated and measured amplitude differences are 1 and $2 \mathrm{~dB}$ at a frequency range of $25-58 \mathrm{GHz}$. The simulated and measured phase differences are from $-178^{\circ}$ to $-187^{\circ}$ and from $-179^{\circ}$ to $-192^{\circ}$ at the frequency range of 20 to $55 \mathrm{GHz}$ (Fig. 6).

\section{B. Trifilar Transformer Dual Balun}

The second test circuit of the trifilar transformer balun is shown in Fig. 7. The design parameters are essentially the same as those of the double spiral transformer dual balun. One port is terminated with $50 \Omega$. The balun size is approximately $400 \times 230 \mu \mathrm{m}^{2}$. The insertion loss of the ideal dual balun is again $6 \mathrm{~dB}$; the simulated and measured insertion loss results are approximately $7-8.5 \mathrm{~dB}$ and $11-9 \mathrm{~dB}$. The simulated

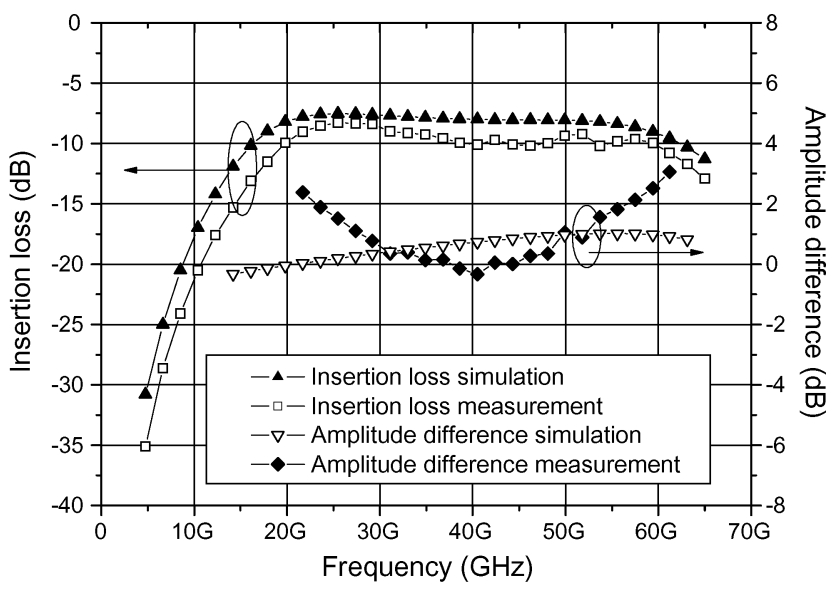

(a)

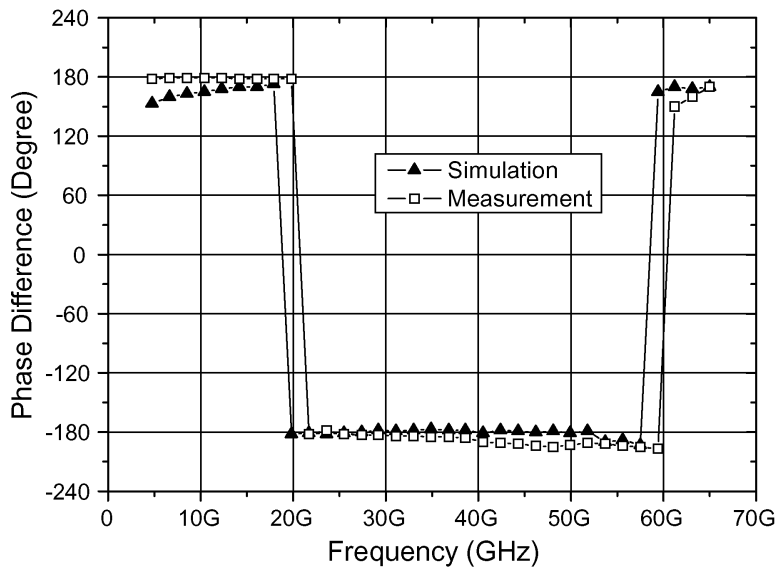

(b)

Fig. 6. Double spiral transformer dual balun simulation and measurement result of: (a) insertion loss and amplitude difference and (b) phase difference.

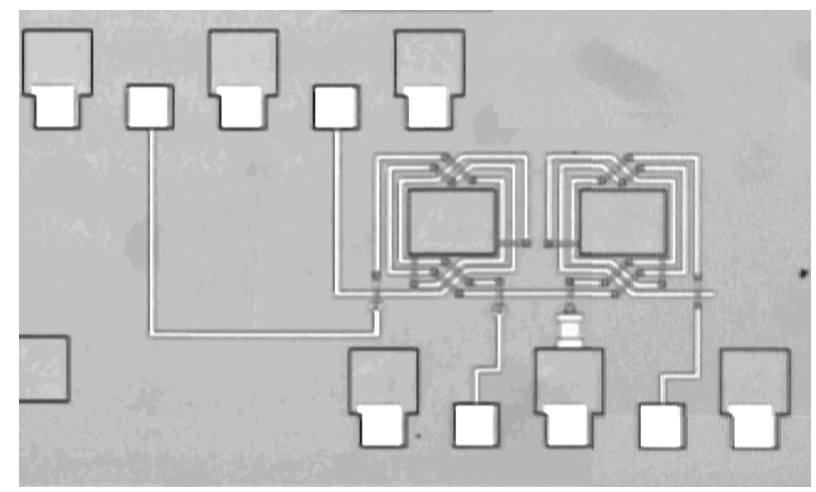

Fig. 7. Chip photograph of the trifilar transformer dual balun. $\left(0.8 \times 0.5 \mathrm{~mm}^{2}\right)$.

and measured amplitude differences are 1 and $2 \mathrm{~dB}$ in the frequency range of $25-58 \mathrm{GHz}$, the simulated and measured phase difference are from $-182^{\circ}$ to $-178^{\circ}$ and from $-183^{\circ}$ to $-171^{\circ}$ in the frequency range of $20-50 \mathrm{GHz}$ (Fig. 8). There are two main differences between these two edge coupled baluns, the first is frequency response. The bandwidth of the double spiral transformer is wider than that of the trifilar transformer, which uses more air bridges. As a result, the capacitive parasitic and more junction discontinuities restrict the performance bandwidth and increase the insertion loss. Figs. 6(a) 


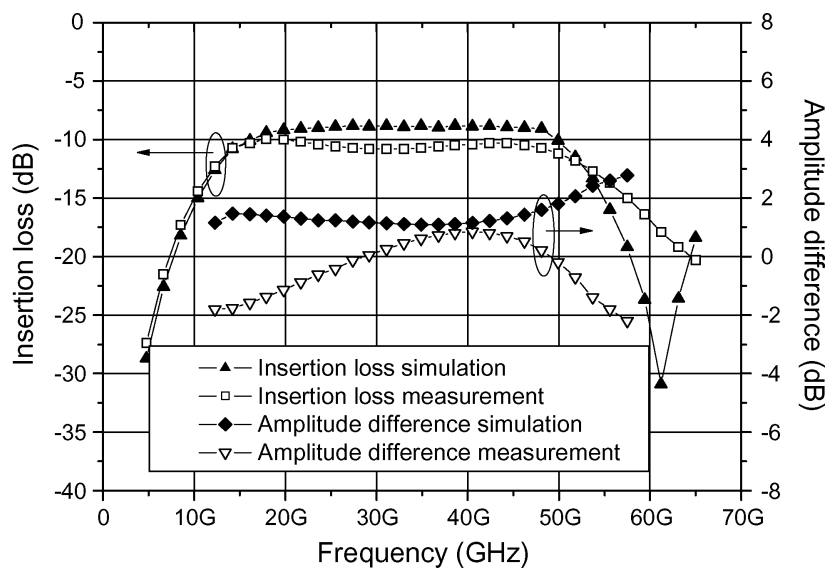

(a)

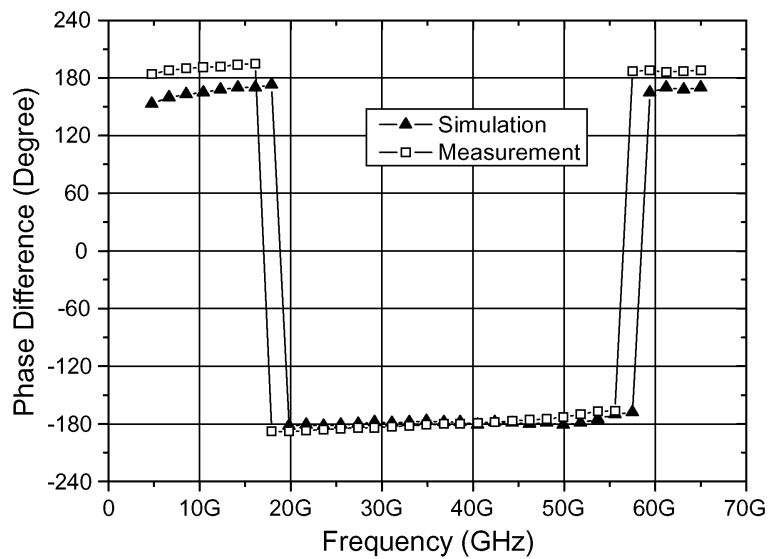

(b)

Fig. 8. Trifilar transformer dual balun simulation and measurement result of: (a) insertion loss and amplitude difference and (b) phase difference.

and 8(a) compare the two; the bandwidth of the double spiral transformer covers $20-60 \mathrm{GHz}$, while the trifilar covers only $20-50 \mathrm{GHz}$. Since the trifilar is a symmetric transformer, the output signal covers more, is almost symmetric, and the phase difference is smaller, but the insertion loss is worse. Another issue is design complexity. The full-wave EM simulator of the double spiral transformer is computationally costly because it is not symmetric, and the adjustment of lines $A$ and $B$ (Fig. 3) is somewhat tedious. In contrast, the trifilar is symmetric so the equal lengths of lines $A$ and $B$ minimizes the adjustment and thus simulation cost.

\section{3-D Transformer Dual Balun}

For the 3-D transformer dual balun, the selected technology is a $0.13-\mu \mathrm{m}$ mixed-signal RF CMOS process. The foundry process provides eight metal layers; the top metal M8 (metal 8) is ultra-thick metal (UTM) and the other metals are thin metals. To reduce the metal loss, two thin metal layers are stacked into one thick metal layer by using a vertical via. The bottom metal (M1) is the reference ground for the dual balun. A side-view diagram is shown in Fig. 3, where a 3- $\mu$ m metal width and a $5-\mu \mathrm{m}$ gap are selected to provide the odd-mode impedance of $28 \Omega$, chosen from Fig. 4 . The test circuit photograph is shown in Fig. 9. The dual balun exhibits wideband frequency performance due to broadside coupling. The simulated and measured

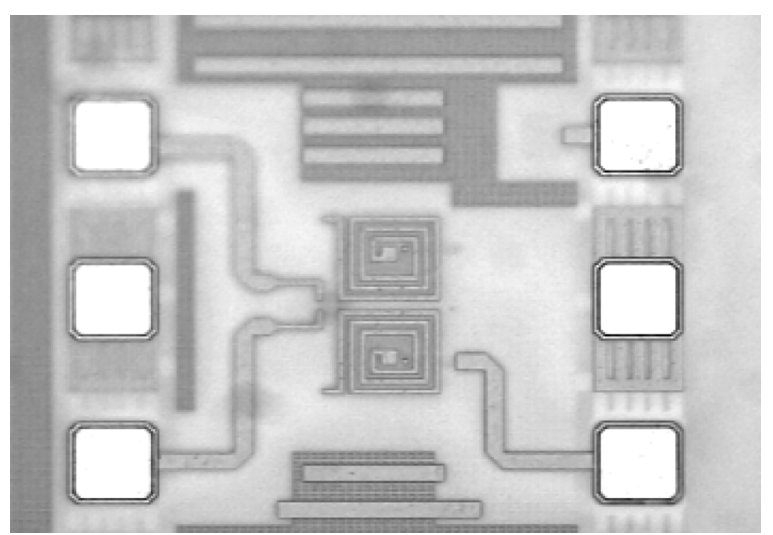

Fig. 9. Chip photograph of the 3-D transformer dual balun. $\left(0.4 \times 0.3 \mathrm{~mm}^{2}\right)$.

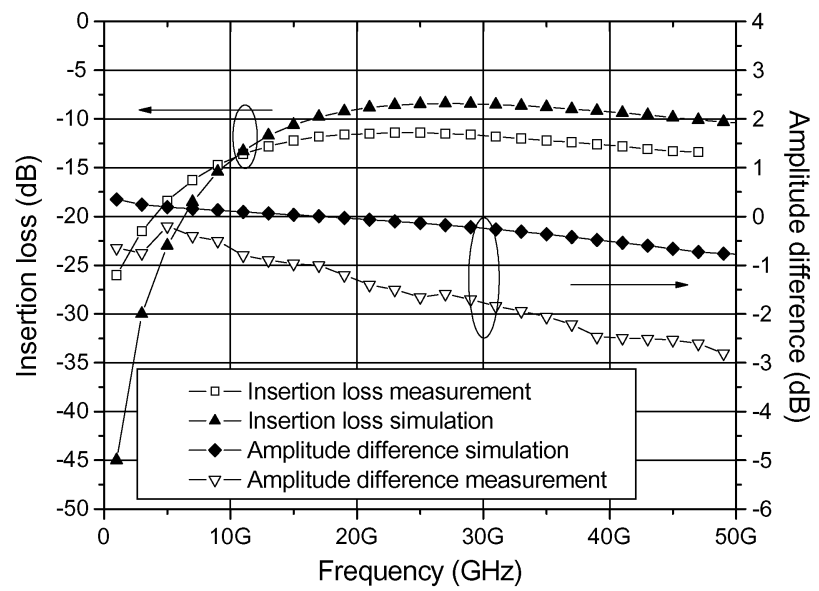

(a)

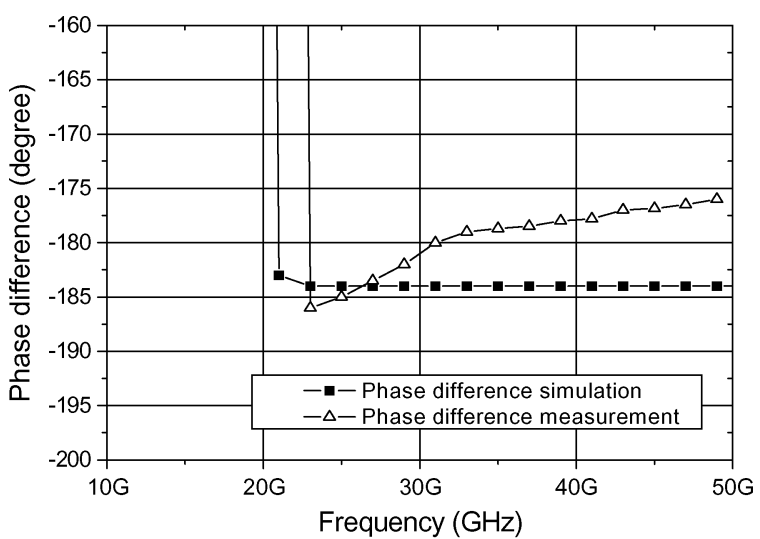

(b)

Fig. 10. 3-D transformer dual balun simulation and measurement result of: (a) insertion loss and amplitude difference and (b) phase difference.

amplitude difference are 1 and $3 \mathrm{~dB}$ at a frequency range of $25-50 \mathrm{GHz}$; the simulated and measured phase differences are from $-184^{\circ}$ to $-182^{\circ}$ and from $-186^{\circ}$ to $-176^{\circ}$ at a frequency range of 20-50 GHz (Fig. 10).

There are some discrepancies between the simulated and measured results in the baluns. For the GaAs-based baluns, the maximum error is $3 \mathrm{~dB}$ within the band of interest, which we think it is due to the electrical parameters in the substrate, such as the conductivity of the metal, dielectric constant, metal 


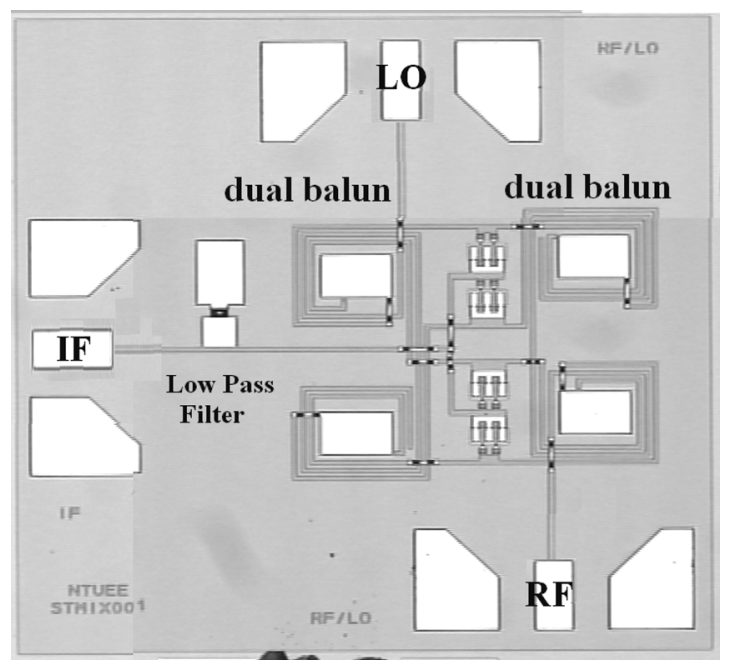

Fig. 11. Chip photograph of star mixer using double spiral transformer dual balun with a chip size of $1 \times 1 \mathrm{~mm}^{2}$.

loss, etc., being entered into the EM simulation tool perhaps not being $100 \%$ accurate, especially in the MMW range. Although the balun in Fig. 3(a) is not as symmetrical as that in Fig. 3(b), the frequency response of the balun in Fig. 3(b) suffers from additional capacitor effect caused by the air-bridge crossover lines. The balun in the CMOS process even has a larger error because the simulation environment of the silicon lossy substrate might have more uncertainties. Moreover, all measurements have limited accuracy, especially these difficult measurements at very high frequencies. Inaccuracies are not necessarily just the EM simulation. Fortunately, due to the DBM architecture, the imbalances of these baluns are not very crucial to the circuit performance.

\section{Star Mixer Design Methodology and Measurement}

Three Marchand dual baluns are proposed in this paper, and these three baluns are separately used in three star mixer designs. The first two mixers are GaAs-based mixers; they are realized using a $0.15-\mu \mathrm{m}$ GaAs pHEMT MMIC process, and the third mixer is a silicon-base resistive mixer realized in a $0.13-\mu \mathrm{m}$ mixed-signal RF CMOS process. These circuits are simulated by a nonlinear circuit simulator (Agilent EEsof Advanced Design System) and all three mixers are measured via probing. Agilent E8241C is used for the LO drive and HP83650B is used for the RF signal input.

\section{A. Double Spiral Transformer Dual Balun Diode Mixer}

The circuit in Fig. 2(b) is designed for a double spiral transformer dual balun mixer. A chip photograph is shown in Fig. 11 with a chip size of $1 \times 1 \mathrm{~mm}^{2}$ and core size of $0.55 \times 0.5 \mathrm{~mm}^{2}$. The diodes of these mixers are all two-finger $20-\mu \mathrm{m}$ devices. The diode is realized by connecting the drain and source of the pHEMT as the cathode, while the gate metallization realizes the anode. The cutoff frequency is near $300 \mathrm{GHz}$. For this diode, a simple model of the parasitic includes a series resistance and a junction capacitance; the series resistance is approximately $9 \Omega$, and the capacitance is approximately $0.05 \mathrm{pF}$. In Fig. 11, the crossover lines are realized using metal air bridges. The shunt

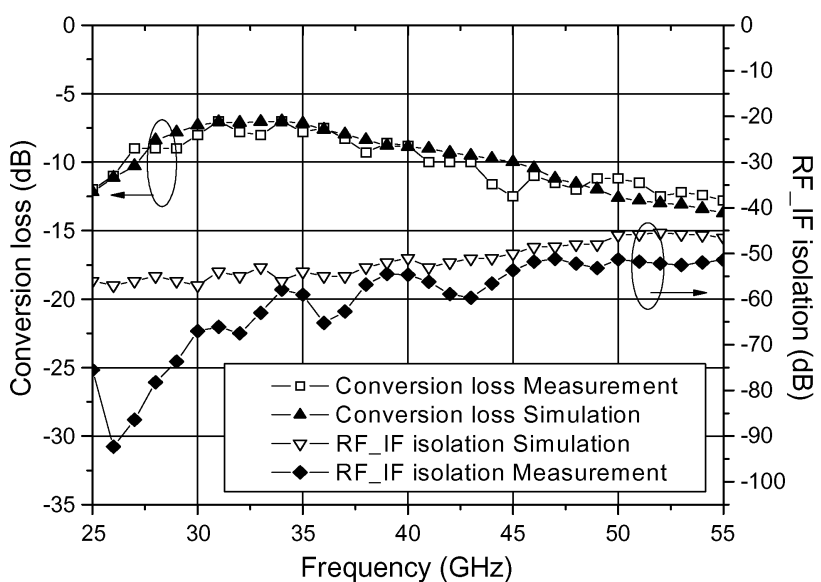

(a)

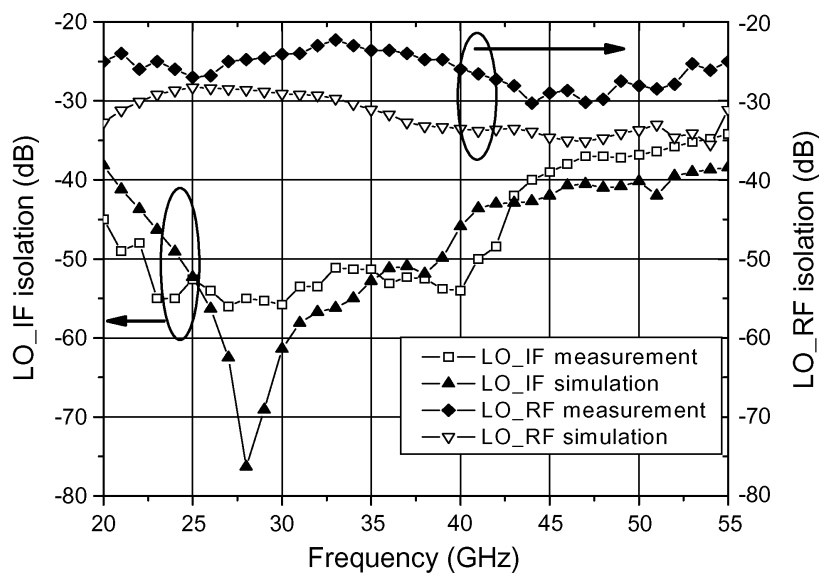

(b)

Fig. 12. Simulated and measured: (a) conversion loss and RF-to-IF isolation and (b) LO-to-RF and LO-to-IF isolations at an IF of $1 \mathrm{GHz}$ for the double spiral transformer dual balun star mixer.

capacitor in the IF port realizes a simple RF filter. Fig. 12 shows the measured and simulated results of this mixer with the RF power of $-15 \mathrm{dBm}$ at an $\mathrm{LO}$ drive of $13 \mathrm{dBm}$. The conversion gain is from -7 to $-12 \mathrm{~dB}$ at a fixed IF of $1 \mathrm{GHz}$, the LO-to-IF and RF-to-LO isolations are better than $40 \mathrm{~dB}$, largely because of the shunt capacitor in the IF port. The LO-to-RF isolation is better than $20 \mathrm{~dB}$ due to the dual balun. The RF input 1-dB compression point is approximately $5 \mathrm{dBm}$ at $30 \mathrm{GHz}$.

\section{B. Trifilar Transformer Dual Balun Diode Mixer}

The circuit shown in Fig. 2(b) is realized as a trifilar transformer dual balun mixer. The chip photograph is shown in Fig. 13 with a chip size of $1 \times 1 \mathrm{~mm}^{2}$ and core size of $0.55 \times 0.4 \mathrm{~mm}^{2}$. Fig. 14 shows the measured and simulated results of this mixer with the RF power of $-15 \mathrm{dBm}$ at an $\mathrm{LO}$ drive of $13 \mathrm{dBm}$. Conversion gain is from -8 to $-12 \mathrm{~dB}$ at a fixed IF of $1 \mathrm{GHz}$; the LO-to-IF and RF-to-LO isolations are better than $40 \mathrm{~dB}$, again because of the shunt capacitor in the IF port. The LO-to-RF isolation is better than $26 \mathrm{~dB}$ due to the dual balun. The RF input 1-dB compression point is $5 \mathrm{dBm}$ at $30 \mathrm{GHz}$. Since the LO-to-RF isolation of the mixer depends on balance performance of the balun, the LO-to-RF isolation of trifilar balun mixer is better than that of the double spiral balun 


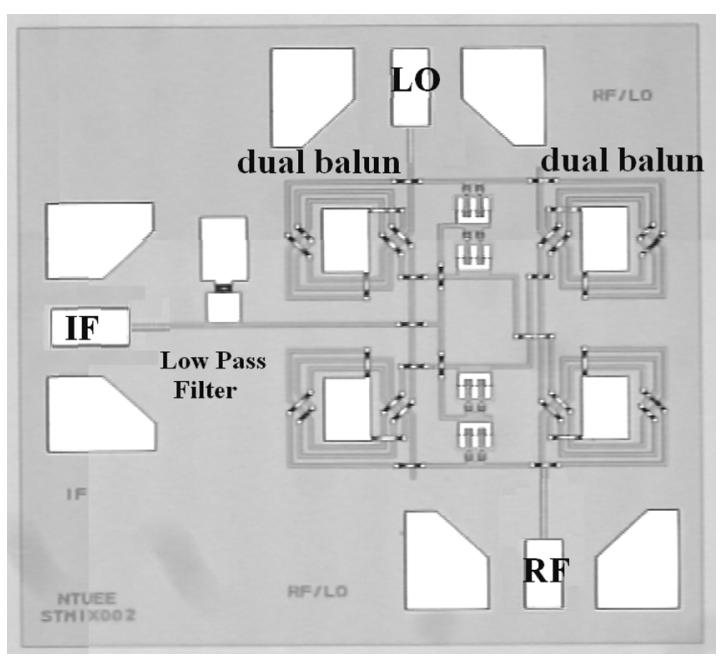

Fig. 13. Chip photograph of star mixer using trifilar transformer dual balun with a chip size of $1 \times 1 \mathrm{~mm}^{2}$.

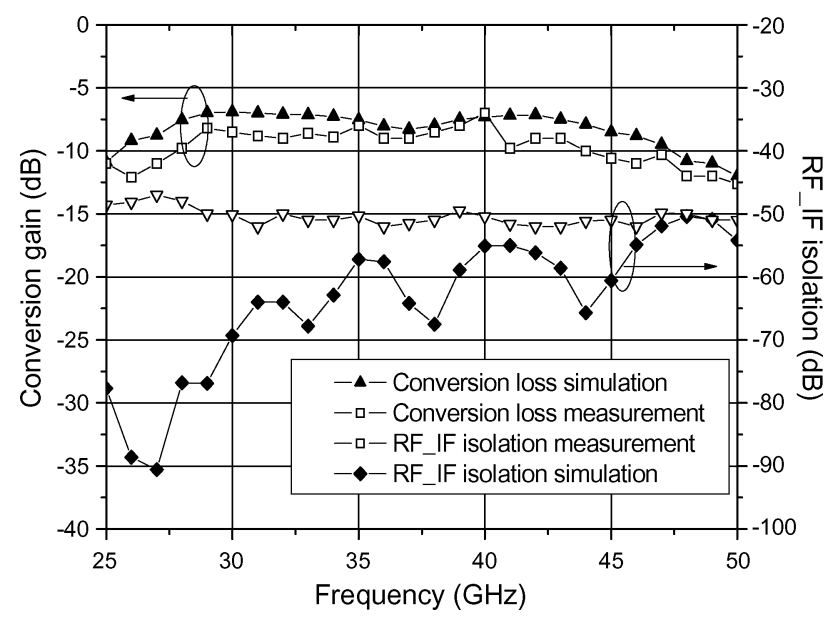

(a)

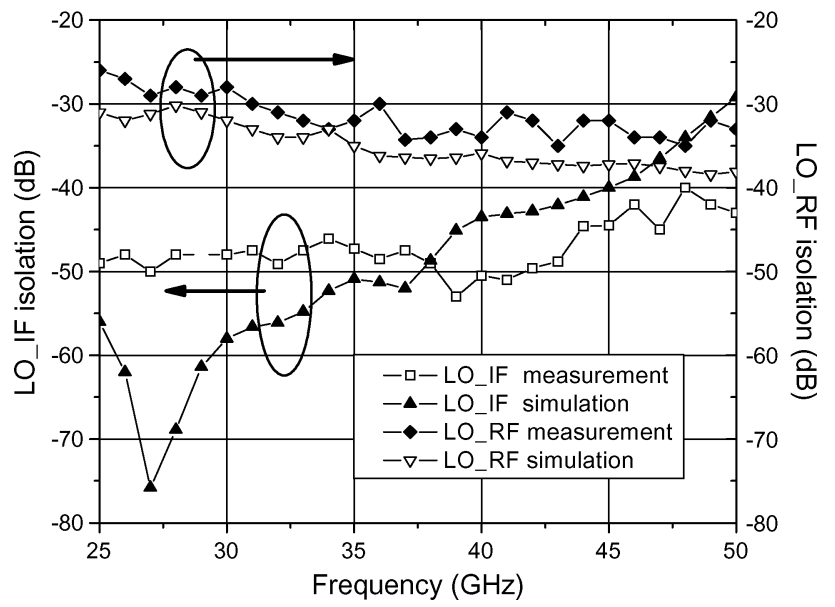

(b)

Fig. 14. Simulated and measured: (a) conversion loss and RF-to-IF isolation and (b) LO-to-RF and LO-to-IF isolations at an IF of $1 \mathrm{GHz}$ for the trifilar transformer dual balun star mixer.

mixer. The isolations of the double spiral balun mixer and trifilar balun mixer are better than 20 and $27 \mathrm{~dB}$, respectively.

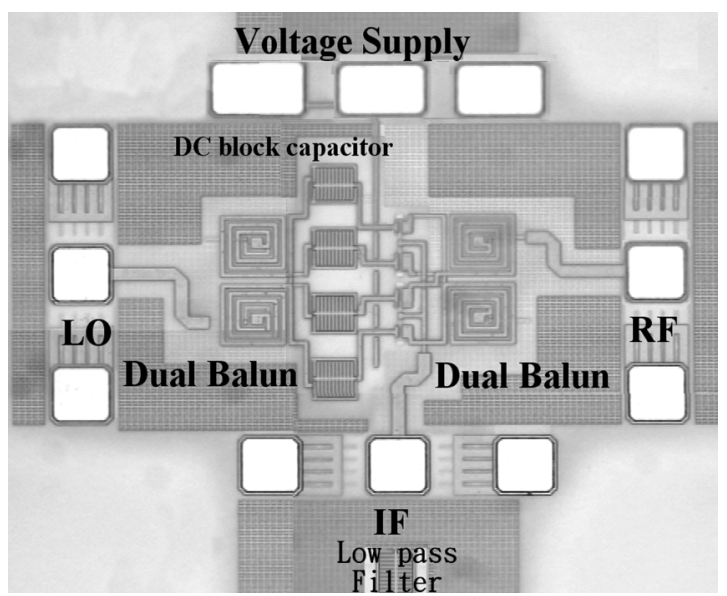

Fig. 15. Chip photograph of star mixer using 3-D transformer dual balun with a chip size of $0.57 \times 0.6 \mathrm{~mm}^{2}$.

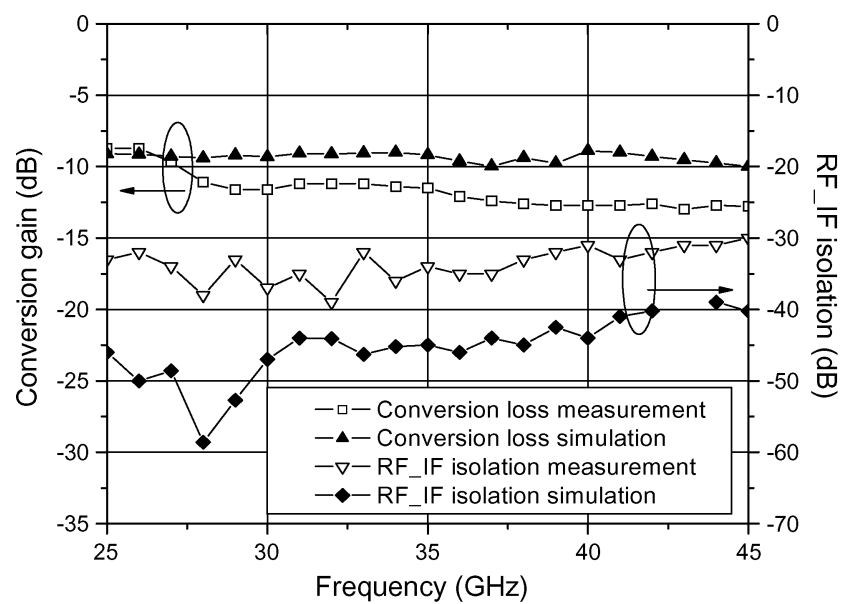

(a)

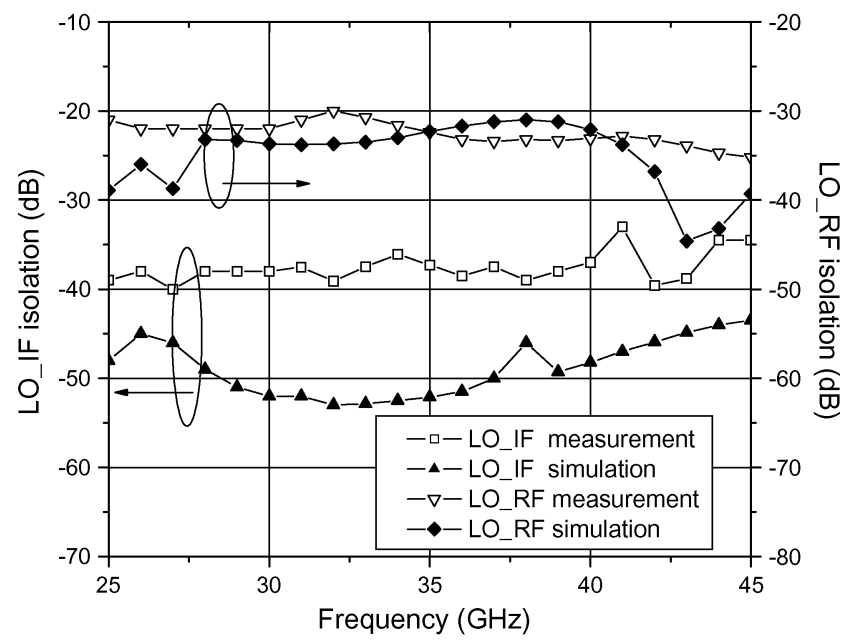

(b)

Fig. 16. Simulated and measured: (a) conversion loss and RF-to-IF isolation and (b) LO-to-RF and LO-to-IF isolations at IF of $1 \mathrm{GHz}$ for the 3-D transformer dual balun star mixer.

Due to the higher insertion loss of the trifilar balun, the LO drive required by the trifilar balun mixer is higher than the double spiral transformer mixer by $1.5 \mathrm{~dB}$. 
TABLE I

PERFoRMANCE COMPARISION OF STAR MiXER

\begin{tabular}{|c|c|c|c|c|c|c|}
\hline & [4] & {$[5]$} & [6] & [7] & This Work & This Work \\
\hline Technology & Package Diode & Schoktty Diode & $\begin{array}{l}0.1-\mu \mathrm{m} \text { pHEMT } \\
\text { connected diode }\end{array}$ & Packaged Diode & $\begin{array}{l}0.15-\mu \mathrm{m} \text { pHEMT } \\
\text { diode }\end{array}$ & $0.13-\mu \mathrm{m}$ CMOS \\
\hline \multirow[t]{2}{*}{ Dual balun } & \multirow[t]{2}{*}{$\begin{array}{l}\text { Mousw's junction } \\
\text { (hybrid circuit) }\end{array}$} & \multirow[t]{2}{*}{ Lump Balun } & \multirow{2}{*}{$\begin{array}{l}\text { Traditional } \\
\text { Dual Marchand } \\
\text { Balun }\end{array}$} & \multirow{2}{*}{$\begin{array}{l}\text { Two identical } \\
\text { Marchand Baluns } \\
\text { series-connected } \\
\text { (hybrid circuit) } \\
\end{array}$} & $\begin{array}{l}\text { Double spiral } \\
\text { transformer }\end{array}$ & \multirow[t]{2}{*}{ 3-D transformer } \\
\hline & & & & & Trifilar transformer & \\
\hline \multirow[t]{2}{*}{ RF Frequency } & \multirow[t]{2}{*}{$\begin{array}{l}1 \sim 35 \mathrm{GHz} \\
\text { (20:1 Bandwidth) }\end{array}$} & \multirow[t]{2}{*}{$\begin{array}{l}1 \sim 6 \mathrm{GHz} \\
(140 \% \text { Bandwidth })\end{array}$} & \multirow{2}{*}{$\begin{array}{l}50 \sim 75 \mathrm{GHz} \\
\text { (40\% Bandwidth) } \\
\text { (Full V-band) }\end{array}$} & \multirow[t]{2}{*}{$\begin{array}{l}1.2 \sim 2.8 \mathrm{GHz} \\
(80 \% \text { Bandwidth) }\end{array}$} & $\begin{array}{l}25 \sim 55 \mathrm{GHz} \\
(65 \% \text { Bandwidth })\end{array}$ & \multirow[t]{2}{*}{$\begin{array}{l}25 \sim 45 \mathrm{GHz} \\
(57 \% \text { Bandwidth) }\end{array}$} \\
\hline & & & & & $\begin{array}{l}25 \sim 50 \mathrm{GHz} \\
\text { (62.5\% Bandwidth) }\end{array}$ & \\
\hline LO Frequency & N.A. & $2 \mathrm{GHz}$ & $58 \mathrm{GHz}$ & $1.2 \sim 2.8 \mathrm{GHz}$ & $40 \mathrm{GHz}$ & $40 \mathrm{GHz}$ \\
\hline \multirow[t]{2}{*}{ Conversion loss } & \multirow[t]{2}{*}{$6 \sim 18 \mathrm{~dB}$} & \multirow[t]{2}{*}{$8 \sim 10 \mathrm{~dB}$} & \multirow[t]{2}{*}{$12 \sim 20 \mathrm{~dB}$} & \multirow[t]{2}{*}{$5 \sim 7 \mathrm{~dB}$} & $7 \sim 12 \mathrm{~dB}$ & \multirow[t]{2}{*}{$8 \sim 13 \mathrm{~dB}$} \\
\hline & & & & & $8 \sim 12 \mathrm{~dB}$ & \\
\hline RF-IF isolation & N.A. & $>30 \mathrm{~dB}$ & N.A. & N.A. & $>30 \mathrm{~dB}$ & $>30 \mathrm{~dB}$ \\
\hline LO-IF isolation & $10-20 \mathrm{~dB}$ & $>20 \mathrm{~dB}$ & N.A. & $23 \mathrm{~dB}$ & $>30 \mathrm{~dB}$ & $>30 \mathrm{~dB}$ \\
\hline \multirow[t]{2}{*}{ LO-RF isolation } & \multirow[t]{2}{*}{$15-30 \mathrm{~dB}$} & \multirow[t]{2}{*}{$20-40 \mathrm{~dB}$} & \multirow[t]{2}{*}{$35 \mathrm{~dB}$} & \multirow[t]{2}{*}{$36 \mathrm{~dB}$} & $>20 \mathrm{~dB}$ & \multirow[t]{2}{*}{$>30 \mathrm{~dB}$} \\
\hline & & & & & $>26 \mathrm{~dB}$ & \\
\hline$\overline{\text { Size }}$ & $\begin{array}{l}370 \text { mil CPS } \\
\lambda / 4 \text { coupled line }\end{array}$ & $\begin{array}{l}0.8 \times 0.8 \mathrm{~mm}^{2} \\
(\lambda / 35 \times \lambda / 35)\end{array}$ & $1.5 \times 1.5 \mathrm{~mm}^{2}$ & $55 \times 56 \mathrm{~mm}^{2}$ & $1 \times 1 \mathrm{~mm}^{2}$ & $0.57 \times 0.6 \mathrm{~mm}^{2}$ \\
\hline Mixer core area & N.A. & N.A. & $(\lambda / 2 \times \lambda / 2)$ & $\begin{array}{l}\text { smaller than } \\
(\lambda / 2 \times \lambda / 2)\end{array}$ & $\begin{array}{l}0.5 \times 0.4 \mathrm{~mm}^{2} \\
(\lambda / 6 \times \lambda / 6.5)\end{array}$ & $\begin{array}{l}0.4 \times 0.3 \mathrm{~mm}^{2} \\
(\lambda / 6.5 \times \lambda / 7)\end{array}$ \\
\hline
\end{tabular}

\section{3-D Transformer Dual Balun Resistive Mixer}

The circuit shown in Fig. 2(c) is realized as the 3-D transformer mixer. The chip photograph is shown in Fig. 15 with a chip size of $0.57 \times 0.6 \mathrm{~mm}^{2}$ and core size of $0.4 \times 0.3 \mathrm{~mm}^{2}$. nMOS and pMOS devices are cascaded to connect with baluns. For the same input impedance, the total width of $32 \mu \mathrm{m}$ is selected for the pMOS device, which is twice that of the NMOS. All transistors operate in a passive mode. The input impedance of the MOS devices can be represented by a simple model, which is a series $20-\Omega$ resistor and $0.05-\mathrm{pF}$ capacitor. Since the silicon subtrate is lossy, higher LO power than those in the GaAs-based mixers is needed. Therefore, dc bias of the transistor near the turn-on region is required. 3- $\mathrm{k} \Omega$ resistors are connected to the gate of the MOS devices and dc pad, and four dc blocking capacitors are series connected to the balun and gate of the MOS devices. Two near-threshold voltages supply $0.5 \mathrm{~V}$ and $-0.5 \mathrm{~V}$ to the gate, and a bias-T is used to connect the IF and dc grounds. Since the devices are biased near the turn-on region, $\mathrm{LO}$ power of only $7 \mathrm{dBm}$ is required. Fig. 16 shows the measured and simulated results of this mixer with the RF power of $-15 \mathrm{dBm}$ and $\mathrm{LO}$ drive of $6 \mathrm{dBm}$. The conversion gain is from -8 to $-14 \mathrm{~dB}$ at a fixed IF of $1 \mathrm{GHz}$, and the LO-to-IF and RF-to-LO isolations are better than $40 \mathrm{~dB}$. The LO-to-RF isolation is better than $30 \mathrm{~dB}$, due to the balance of dual balun; the output power level is approximately $-30 \mathrm{dBm}$.

It is observed that the imbalances of the baluns are up to 3-dB differences (Figs. 6, 8, and 10), but the LO-to-RF isolations of the mixers are still acceptable due to the natures of the double-balanced mixers. We have seen this consistently with other star mixers. Regarding the LO-to-IF and RF-to-IF isolation since there is a low-pass filter with 3-dB cutoff frequency of approximately $7 \mathrm{GHz}$ with over a $30-\mathrm{dB}$ rejection above $25 \mathrm{GHz}$ in the IF path, the isolations for 1-GHz IF are taken care of by this low-pass filter.

Table I compares a number of published results of star mixer designs. In this table, an ultra-broadband mixer is proposed by Chang et al. [4]; however, the mixer still needs two $\lambda / 4$ CPS lines, and a special absorber or suspended substrate for ultrabroadband performance. Therefore, it is difficult to realize in MMICs. Wideband performance and full $V$-band performance using a traditional balun and novel hybrid Marchand dual balun and are presented by Yeom and Ko [6] and Kim et al. [7] with a complete analysis of drain-source and drain-gate connected pHEMT diode performance is discussed [6]; however, the circuits still need $\lambda / 2$ baluns and lead to large areas. A miniature mixer using lumped-element baluns is proposed by Chiou et al. [5], but this circuit cannot be realized in the MMW range because of the low self-resonant frequency of the lumped baluns. Therefore, the novel proposed star mixers in this paper indeed demonstrate good performances with smallest size compared to the previously reported star mixers.

\section{CONCLUSION}

In this paper, three novel small-size and high-performance star mixers have been proposed; the LO power is 13 and $15 \mathrm{dBm}$ and $7 \mathrm{dBm}$ for GaAs- and silicon-based mixers, respectively. 
These three mixer cores occupy only $500 \times 500 \mu \mathrm{m}^{2}(\lambda / 6 \times \lambda / 6)$ or smaller. Compared to the traditional star mixers, which typically need a core area of $\lambda / 2 \times \lambda / 2$, a size reduction of almost $80 \%$ is achieved. Among these mixers, the 3-D balun mixer provides the best isolations and smallest chip size, but requires a multilayer MMIC process. In the GaAs-based mixers, higher LO-to-RF isolation of the trifilar balun mixer can be achieved, with the requirement of a higher $\mathrm{LO}$ drive.

\section{ACKNOWLEDGMENT}

The authors would like to thank the staff of WIN Semiconductors, Tiawan, R.O.C., the Tiawan Semiconductor Manufacturing Company (TSMC), Tiawan, R.O.C., and the Chip Implementation Center (CIC) Taiwan, R.O.C., and P. S. Wu, M. F. Lei, C. H. Wang, C. F. Chao, T. P. Wang, all with National Taiwan University, Taiwan, R.O.C., and Prof. H. K. Chiou and Prof. H. Y. Chang, both with National Central University, Taiwan, R.O.C., for their helpful suggestions.

The chips used in this study were fabricated by WIN Semiconductors and TSMC through the CIC.

\section{REFERENCES}

[1] S. A. Maas, Microwave Mixers, 2nd ed. Norwood, MA: Artech House, 1993.

[2] S. Basu and S. A. Maas, "Design and performance of a planar star mixer," IEEE Trans. Microw. Theory Tech., vol. 41, no. 11, pp. 2028-2030, Nov. 1993.

[3] Y. I. Ryu, K. W. Kobayashi, and A. K. Oki, "A monolithic broadband doubly balanced EHF HBT star mixer with novel microstrip baluns," in IEEE Microw. Millimeter-Wave Monolithic Circuits Symp. Dig., 1995, pp. 119-122.

[4] C. Y. Chang, C. W. Tang, and D. C. Niu, "Ultra-broad-band doubly balanced star mixers using planar Mouw's hybrid junction," IEEE Trans. Microw. Theory Tech., vol. 41, no. 6, pp. 1077-1085, Jun. 2001.

[5] H. K. Chiou, Y. R. Juang, and H. H. Lin, "Miniature MMIC star double balanced mixer using lumped dual balun," Electron. Lett., vol. 33, no. 6, pp. 503-505, Mar. 1997.

[6] K. W. Yeom and D. H. Ko, "A novel 60-GHz monolithic star mixer using gate-drain-connected pHEMT diodes," IEEE Tran. Microwave Theory Tech., vol. 53, no. 7, pp. 2435-2440, Jul. 2005.

[7] S. S. Kim, J. H. Lee, and K. W. Yeom, "A novel planar dual balun for doubly balanced star mixer," IEEE Microw. Wireless Compon. Lett., vol. 14, no. 9, pp. 440-442, Sep. 2004.

[8] J. R. Long, "monolithic transformers for silicon RF IC design," IEEE J. Solid-State Circuits, vol. 35, no. 9, pp. 1368-1382, Sep. 2000.

[9] P. S. Wu, C. H. Wang, T. W. Huang, and H. Wang, "Compact and broadband millimeter-wave monolithic transformer balanced mixers," IEEE Trans. Microw. Theory Tech., vol. 53, no. 10, pp. 3106-3114, Oct. 2005.

[10] J. R. Long, "A low-voltage 5.1-5.8-GHz image-reject down converter RF IC," IEEE J. Solid-State Circuits, vol. 35, no. 9, pp. 1320-1328, Sep. 2000.

[11] V. Trifunovic and B. Jokanovic, "Star mixer with high port-to-port isolation," Electron. Lett., vol. 32, no. 24, pp. 2251-2252, Nov. 1996.

[12] J. Staudingerm and M. Friesen, "Fully integrated double balanced MMIC mixer using a star arrangement of diodes for extended IF performance," in IEEE MTT-S Int. Microw. Symp. Dig., Jun. 1-5, 1992, vol. 3, pp. 1163-1166.

[13] B. R. Hallford, "100-mWatt output up converter using Schottky diodes," in IEEE MTT-S Int. Microw. Symp. Dig., 1979, pp. 492-494.

[14] N. Marchand, "Transmission-line conversion transformers," Electronics, vol. 17, pp. 142-145, Dec. 1944.

[15] R. B. Mouw, "A broadband hybrid junction and application to the star modulator," IEEE Trans. Microw. Theory Tech., vol. MTT-16, no. 11, pp. 154-161, Nov. 1968.
[16] I. Nam, B. Kim, and K. Lee, "CMOS RF amplifier and mixer circuits utilizing complementary Characteristics of parallel combined nMOS and pMOS devices," IEEE Trans. Microw. Theory Tech., vol. 53, no. 5, pp. 1662-1671, May 2005.

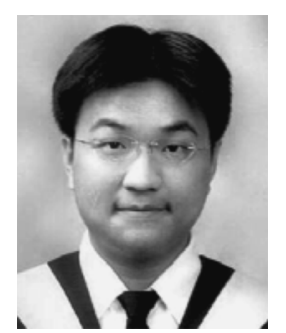

Che-Chung Kuo was born in Taipei, Taiwan, R.O.C., in 1980. He received the M.S. degree in electric engineering from National Center University, Chung-Li, Taiwan, R.O.C., in 2005, and is currently working toward the Ph.D. degree in communication engineering at National Taiwan University, Taipei, Taiwan, R.O.C.

His research interests include microwave circuits designs and microwave circuit system-on-package integration.

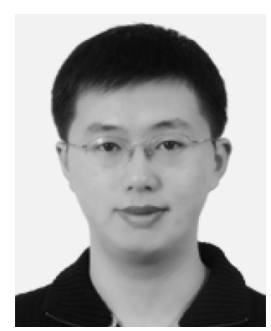

Chun-Lin Kuo was born in Kaohsiung, Taiwan, R.O.C., in 1983. He received the M.S. degree in communication engineering from National Taiwan University, Taipei, Taiwan, R.O.C., in 2007.

His research interests include microwave and MMW circuits designs.

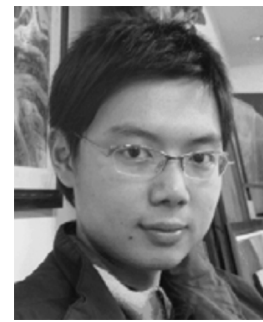

Che-Jia Kuo was born in Yunlin, Taiwan, R.O.C., in 1983. He received the B.S. degree in electrical engineering from National Taiwan University, Taipei, Taiwan, R.O.C., in 2005, and is currently working toward the Ph.D. degree at the Graduate Institute of Communication Engineering, National Taiwan University.

His research interests include monolithic microwave/MMW circuit design.

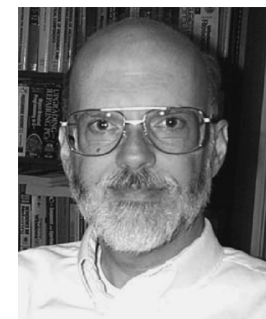

Stephen A. Maas (M'74-SM'89-F'93) received the B.S. and M.S. degrees from the University of Pennsylvania, Philadelphia, in 1971 and 1972, respectively, and the Ph.D. degree from the University of California at Los Angeles (UCLA), in 1984, all in electrical engineering.

Since then, he has been involved in research, design, and development of low-noise and nonlinear microwave circuits and systems with the National Radio Astronomy Observatory (where he designed the receivers for the Very Large Array), Hughes Aircraft Co., TRW, the Aerospace Corporporation, and the Department of Electrical Engineering, UCLA. He was subsequently an engineering consultant and founded Nonlinear Technologies Inc., Long Beach, CA, a consulting company, in 1993. He is also Chief Scientist of Applied Wave Research Inc. (AWR Inc.), El Segundo, CA. He authored Microwave Mixers (Artech House, 1986 and 1992), Nonlinear Microwave Circuits (Artech House, 1988 and 2003), The RF and Microwave Circuit Design Cookbook (Artech House, 1998), and Noise in Linear and Nonlinear Circuits (Artech House, 2005).

Dr. Maas was the editor-in-chief of the IEEE TRANSACTIONS ON MICROWAVE THEORY AND TECHNIQUES (1990-1992). From 1990 to 1993, he was an IEEE Microwave Theory and Techniques Society (IEEE MTT-S) Administrative Committee (AdCom) member and publications chairman. He was the recipient of the 1989 Microwave Prize for his work on distortion in diode mixers and the 2002 IEEE MTT-S Application Award. 


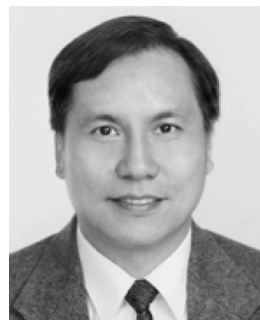

Huei Wang (S'83-M'87-SM'95-F'06) was born in Tainan, Taiwan, R.O.C., on March 9, 1958. He received the B.S. degree in electrical engineering from National Taiwan University, Taipei, Taiwan, R.O.C., in 1980, and the M.S. and Ph.D. degrees in electrical engineering from Michigan State University, East Lansing, in 1984 and 1987, respectively.

During his graduate study, he was engaged in research on theoretical and numerical analysis of EM radiation and scattering problems. He was also involved in the development of microwave remote detecting/sensing systems. In 1987, he joined the Electronic Systems and Technology Division, TRW Inc. He has been an MTS and Staff Engineer responsible for MMIC modeling of computer-aided design (CAD) tools, MMIC testing evaluation, and design and became the Senior Section Manager of the Mil-
limeter-Wave (MMW) Sensor Product Section, RF Product Center. In 1993, he visited the Institute of Electronics, National Chiao-Tung University, Hsinchu, Taiwan, R.O.C., to teach MMIC-related topics. In 1994, he returned to TRW Inc. In February 1998, he joined the faculty of the Department of Electrical Engineering, National Taiwan University, as a Professor. He is currently the Director of the Graduate Institute of Communication Engineering, National Taiwan University.

Dr. Wang is a member of Phi Kappa Phi and Tau Beta Pi. He was the recipient of the Distinguished Research Award of the National Science Council, R.O.C. (2003-2006). In 2005, he was elected as the first Richard M. Hong Endowed Chair Professor of National Taiwan University. He has been appointed an IEEE Distinguished Microwave Lecturer for the 2007-2009 term. He was the recipient of the 2007 Academic Achievement Award presented by the Ministry of Education, R.O.C. 